\title{
The PTPN22* C1858T functional polymorphism is associated with susceptibility to inflammatory polyarthritis but neither this nor other variants spanning the gene is associated with disease outcome
}

\author{
H Naseem, W Thomson, A Silman, J Worthington, D Symmons, A Barton
}

ARC Epidemiology Unit, University of Manchester, UK

Correspondence to:

A Barton ARC Epidemiology Unit, University of Manchester, Oxford Road, Manchester, M13 9PT, UK; anne.barton@ manchester.ac.uk

Accepted 1 July 2007 Published Online First 31 July 2007

\section{UNLCKII}

This paper is freely available online under the BMJ Journals unlocked scheme, see http:// ard.bmj.com/info/unlocked.dtl

\begin{abstract}
Background: The PTPN22 gene has been widely confirmed as a susceptibility gene for rheumatoid arthritis (RA) in populations of Northern European descent. The aim of the current study was to explore the role of variants spanning the PTPN22 gene in determining susceptibility to and outcome of inflammatory polyarthritis (IP).
\end{abstract}

Patients and methods: Single nucleotide polymorphism (SNP) variants spanning the gene were genotyped using the Sequenom MassArray platform and tested, firstly for their association with susceptibility to IP. Genotype frequencies were compared between new onset IP cases $(n=843)$ and population controls $(n=471)$. Secondly, a within-cohort analysis was performed testing each variant for association with a number of clinical outcome measures reflecting disease severity including radiological erosions, physical function, measured using the Health Assessment Questionnaire (HAO) score, and disease activity at defined time-points following disease presentation.

Results: A significant association between carriage of the PTPN22*1858T allele and IP (odds ratio (OR) $=1.4$ $(95 \% \mathrm{Cl} 1.1-1.9), p=0.02)$ was observed. The strength of the effect was similar in the RA subgroup (OR $=1.4$ (95\% Cl 1.0-1.9), $p=0.05)$. No association between IP susceptibility and any of the other SNPs was detected. No association was detected for any of the SNPs tested, including the PTPN22*C1858T polymorphism, for either erosive status, Larsen score by 5 years or other markers of clinical outcome.

Conclusion: The PTPN22*C1858T polymorphism is associated with susceptibility to IP, but we have found no evidence for association of this or other variants spanning the gene with clinical outcome measures.

A considerable genetic contribution to rheumatoid arthritis (RA) is demonstrated by both twin and family studies. ${ }^{1}$ The strongest association to date has been found with the HLA-DRB1 gene and, in particular, with alleles that code for a consensus amino acid sequence within the third hypervariable region of the DRB1 chain (the shared-epitope). More recently, progress has been made in identifying other RA susceptibility genes (reviewed in Orozco et al). ${ }^{2}$ The most convincing association has been demonstrated with a functional single nucleotide polymorphism (SNP) mapping to the lymphoid tyrosine phosphatase, PTPN22, gene $\left(\right.$ PTPN22 ${ }^{*}$ C1858T). ${ }^{3}$ This finding has been widely and consistently replicated in populations of Northern European descent. ${ }^{4}$

The PTPN22 gene maps to chromosome $1 \mathrm{p} 13$ and codes for a tyrosine phosphatase that is involved in immune regulation as a negative regulator of $\mathrm{T}$ cell activity. The polymorphism results in the substitution of the wild-type, arginine, with a variant, tryptophan, amino acid at codon 620 (R620W). Recent work suggests that the polymorphism may lead to a "gain-of-function" as the protein has been shown to be changed into a more active tyrosine phosphatase in the presence of the tryptophan variant. ${ }^{5}$ It is hypothesised that reduced T-cell signalling may occur as a result and, in turn, this may enable auto-reactive $\mathrm{T}$ cells to escape deletion during thymic selection and thus persist in the circulation to become activated at a later stage. Although the primary association is with the PTPN22 ${ }^{*} \mathrm{C} 1858 \mathrm{~T}$ polymorphism, evidence for independent association of three other SNPs mapping within the gene with susceptibility to RA has since been reported but this finding has not been replicated in two subsequent studies. ${ }^{6-8}$

In the clinical setting, the greatest utility for genetic markers would be if they led to the identification of a marker that could predict, at the earliest stages of inflammatory polyarthritis (IP), which patients will proceed to develop more severe disease. This could help to identify a subgroup of patients that could be targeted for aggressive therapy, while sparing those least likely to develop severe disease from the potential side effects associated with such drug therapy. While both the shared epitope and the PTPN22 ${ }^{*} \mathrm{C} 1858 \mathrm{~T}$ allele are certainly associated with RA, the question of whether these associations are primarily with susceptibility or severity has proved difficult to determine because many previous studies have investigated cross-sectional series of hospital-based cases that often represent the severe end of the spectrum of disease. Ideally, such studies should be performed in inception cohorts of patients followed prospectively. In an unselected series of patients presenting to primary care with early IP and followed prospectively as part of the Norfolk Arthritis Register (NOAR), we have previously shown that the shared epitope is more strongly associated with severity markers such as radiological erosions than with susceptibility. 
Previous studies of the PTPN22*C1858T SNP have revealed stronger associations in patients with a family history of disease and in patients with a younger age at onset suggesting that this polymorphism, too, may be associated more with severity than susceptibility. ${ }^{310}$ However, these studies were cross-sectional in nature and subject to the methodological biases outlined. Only one study has examined an inception cohort followed prospectively. ${ }^{11}$ They reported no association with remission or rates of RA joint destruction but an association between PTPN22*1858T risk allele and autoantibody production was noted.

We have used the opportunity afforded by the NOAR cohort to address the question of firstly, whether other variants spanning the PTPN22 gene are associated with susceptibility to IP and, secondly, whether any is associated with outcome of disease.

\section{MATERIALS AND METHODS \\ Study design}

SNP variants spanning the PTPN22 gene were tested firstly for their association with susceptibility to IP by comparing genotype and allele frequencies between new onset IP cases with population controls. Secondly, a within-cohort analysis was performed testing each variant for association with a number of clinical outcome measures reflecting disease severity including presence of radiological erosions, physical function as measured using the Health Assessment Questionnaire (HAQ) score and disease activity at defined time-points following disease presentation. ${ }^{12}$

\section{Study population}

Cases were recruited from NOAR, a primary care-based cohort of subjects with new onset IP. ${ }^{13}$ In brief, NOAR aims to recruit all new adult attendees at primary care with IP, defined as swelling of two or more joints, lasting $>4$ weeks, after exclusion of diagnoses other than undifferentiated IP, post-viral IP, RA or psoriatic arthritis. Subjects are assessed by trained metrologists, within 2 weeks of referral, using a standardised approach. Data gathered included joint counts for swelling and tenderness. Blood was taken for serum analysis (rheumatoid factor (RF), C-reactive protein (CRP) and anti-cyclic citrinulated peptide (CCP) testing) and DNA extraction. Subjects were followed up annually thereafter. ${ }^{13}$ The 1987 criteria for RA were applied at every visit and subjects classified as having RA if they had cumulatively satisfied the criteria by that time point. ${ }^{14}$ At baseline and annually thereafter the subjects completed the eight-domain HAO. Radiographs of the hands and feet were obtained at 1 and/or 5 years. The criteria for requesting radiographs and the scoring process have been published previously. ${ }^{15}$ The radiographs were read by two readers (with arbitration by a third where necessary) using the Larsen method. ${ }^{16}$ For the purposes of this study, consecutive patients recruited between 1990 and 1994 who had a DNA sample available were selected.

Data for unmatched control individuals recruited from Norfolk general practitioners' records or from blood donors were available and have been reported previously. ${ }^{7}$ All subjects were of UK Caucasoid origin. The study was conducted with ethical approval (LREC 2003-075, MREC 99/8/84) and all patients gave their informed consent.

\section{Serological tests}

Baseline serum samples were assayed for RF was using a latex method and a titre of $\geqslant 1: 40$ was regarded as positive. CRP was measured using nephelometry (http://www.bmglabtech.com/ templates/applications). Anti-CCP testing was performed using the Axis-Shield ${ }^{\circledR}$ DIASTAT ${ }^{\mathrm{TM}}$ kit according to manufacturer's instructions and a result of $>5 \mathrm{U} / \mathrm{ml}$ was regarded as positive (Axis-Shield, Dundee, UK).

\section{Genotyping}

Thirteen SNPs spanning the PTPN22 gene were selected for investigation. These included the PTPN22 *C1858T polymorphism, seven tagging SNPs and two of three potentially functional polymorphisms identified by Carlton et al. ${ }^{6}$ The third SNP, rs3789604 (SNP 37), was not selected for genotyping because of the strong LD exhibited between this and another of the selected SNPs, rs3811021 (SNP 36). Genotyping was undertaken using the Sequenom ${ }^{\circledR}$ iPLEX platform ${ }^{\mathrm{TM}}$, according to the manufacturer's instructions ${ }^{17}$ (http://www.sequenom.com). Samples were plated onto $2 \times 384$-well plates. Duplicate samples and negative controls were included to check genotyping quality. For the PTPN22 ${ }^{*}$ C1858T (rs2476601, R620W) SNP, additional samples from the cohort had been genotyped inhouse previously and that data was included in the current analysis. Only SNPs that were successfully genotyped in $>90 \%$ samples were included in the analysis.

\section{Statistical analysis}

Genotype and allele carriage frequencies for each SNP were compared between cases and controls using the $\chi^{2}$ test for trend and odds ratios (OR) with 95\% confidence interval (CI), respectively. Given the linkage disequilibrium known to exist across the gene, logistic regression was undertaken conditioned on the effect of the associated SNP, rs2476601 (R620W). Estimated haplotypes were constructed in Haploview v3.2 $2^{18}$ and frequencies compared between cases and controls.

For the within-cohort analysis of association with disease severity, a number of measures were assessed reflecting disease activity (CRP at baseline, swollen joint count at baseline and at 5 years following presentation), severity (presence of erosions by 1 and 5 years, Larsen score by 1 and 5 years, presence of antiCCP antibodies and RF at baseline) and disability (HAQ score). Categorical data (erosions by 1 and 5 years, anti-CCP and RF) were analysed using the $\chi^{2}$ test while continuous variables were analysed using the Mann-Whitney $U$ test. All analyses were performed in the cohort as a whole and in the subset of patients fulfilling American College of Rheumatology (ACR) criteria for RA by 5 years using STATA ${ }^{\circledR}$ (StataCorp LP, College Station, Texas, USA).

For the PTPN22*C1858T SNP, there was a clear a priori hypothesis for testing for association with IP and so no correction was made for multiple testing. However, for the other SNPs tested, the prior probability of detecting association was small and a Bonferroni correction of 12 was applied to correct for number of SNPs tested.

\section{RESULTS \\ Subjects}

In total, 1098 patients were recruited to NOAR between 1990 and 1994 and a DNA sample was available from 843 of them. However, for the majority of SNPs, only 750 samples were tested. Clinical characteristics of the cases are detailed in table 1 and are similar to those reported previously from the NOAR cohort. ${ }^{14}$ The median age at onset of IP was 53 (interquartile range (IOR) 40-65), 539 (64\%) were female and 79.4\% had been referred to hospital (secondary care) following onset of their 
arthritis. Of the 448 controls where data were available, 54\% were female and the median age at the time of sample donation was 66 (interquartile range (IOR) 42-75). This data has been published previously. All cases and controls were of UK white Caucasian ethnicity.

\section{Genotyping}

Genotype frequencies for one SNP (ss38346943) deviated from Hardy-Weinberg equilibrium $(p<0.01)$ in the control population and it was excluded from the susceptibility analysis. One variant (ss38346944) was not tested in the control samples, and hence investigation of this marker was restricted to the analysis of clinical outcome.

\section{Association with IP susceptibility}

Genotype distributions differed significantly at the PTPN22*C1858T polymorphism between IP cases and controls $(\mathrm{p}=0.02) \quad$ (table 2). In addition, there was a significant association between carriage of the PTPN22*1858T allele and IP (odds ratio $(O R)=1.42(95 \%$ CI 1.06-1.90), $p=0.02)$. The strength of the effect was similar in the RA subgroup $(\mathrm{OR}=1.38$ (95\% CI 1.01-1.89), $\mathrm{p}=0.04)$ and the IP cohort as a whole. No association between IP and any of the other SNPs was detected either when analysed by genotype (table 2) or by allele. Stratifying the cohort for the fulfilment of ACR criteria for RA by 5 years did not alter these conclusions (data not shown).

When the analysis was performed using logistic regression to adjust for the genotype at the rs2476601 (R620W) SNP, another SNP showed evidence for association with susceptibility: rs1217388 (SNP 32; uncorrected $p=0.003$ ). However, the only haplotype occurring significantly more frequently in cases than controls was the haplotype carrying the risk allele at rs2476601 (R620W) (12.4\% cases, 9.3\% controls, $p=0.03)$. When chromosomes carrying the risk allele were excluded, no evidence for association of the remaining haplotypes with RA was found. Furthermore, haplotypes carrying the risk allele at rs1217388 (SNP 32), showed no difference in frequency between cases and controls.

\section{Association with clinical outcome measures}

No association was detected for any of the SNPs tested, including the PTPN22*C1858T polymorphism, with either

Table 1 Clinical data for subjects with inflammatory polyarthritis (IP)

\begin{tabular}{lr}
\hline Characteristic & Cases $(\mathbf{n}=\mathbf{8 4 3})$ \\
\hline RF positive, n (\%): & $202 / 773(26 \%)$ \\
$\quad$ Baseline & $159 / 476(33 \%)$ \\
Erosive, $n$ (\%): & $202 / 487(41 \%)$ \\
By 1 year & $181 / 682(27 \%)$ \\
By 5 years & $519 / 769(67 \%)$ \\
Anti-CCP positive at baseline, $n$ (\%) & $4(1-10)$ \\
ACR criteria fulfilled by 5 years, $n(\%)$ & $0(0-1)$ \\
Median swollen joint count (IQR): & \\
At baseline & $0.75(0.25-1.38)$ \\
At 5 years & $0.25(0.25-1.5)$ \\
Median HAO score (IQR): & $5(0-15)$ \\
At baseline & \\
At 5 years & \\
Median CRP at baseline (IQR) & \\
\hline The denominator details the number of subjects where information was available. \\
ACR, American College of Rheumatology; CCP, cyclic citrinulated peptide; HAO, Health \\
Assessment Questionnaire; IQR, interquartile range; RF, rheumatoid factor.
\end{tabular}

erosive status or Larsen score by 5 years (table 3 ). A trend for association of the Larsen score by 5 years was noted with SNP rs1217413 (SNP35) but this was not significant when a Bonferroni correction was applied. Furthermore, no association with Larsen score at 1 year or with any other clinical outcome measure was detected (data not shown). None of the other SNPs tested demonstrated statistical evidence for association either in the IP cohort as a whole (table 3 ) or in the subgroup satisfying ACR criteria for RA by 5 years (data not shown). No association was detected with other markers of outcome tested such as HAQ score at presentation or by 5 years, swollen and tender joint counts at presentation or by 5 years or CRP at baseline for any of the SNPs spanning the gene (data not shown).

\section{Association with autoantibody status}

There was a significant association between the variant allele of the PTPN22 ${ }^{*}$ C1858T (rs2476601) SNP and baseline anti-CCP status in the RA subgroup ( $p=0.003$ ) (table 4$)$.

\section{DISCUSSION}

In this unselected primary care-based inception cohort of patients, we have confirmed the association between the PTPN22 ${ }^{*}$ C 1858 T polymorphism and susceptibility to IP but found no association with severity using a number of clinical outcome markers. We have also failed to find any association between disease susceptibility or severity and other polymorphisms spanning the gene.

The association of the PTPN22*1858T variant with susceptibility to RA has been widely replicated in white European populations. ${ }^{4}$ This polymorphism has also been found to be associated with susceptibility in two other inception cohorts with early disease (OR 1.3 and 1.5). ${ }^{11}{ }^{19}$ The effect size detected in the current study $(\mathrm{OR}=1.4)$ is in keeping with those reports and suggests that the variant confers a modest increase in risk.

One explanation for the lower effect sizes detected in studies of early disease compared to established series of RA patients may be that the PTPN22*C1858T variant is associated with disease severity as well as susceptibility. An inception cohort, such as NOAR, followed prospectively is required to dissect these issues. The NOAR cohort is representative of patients with new-onset IP seen in general practice. A total of $79.4 \%$ were referred to hospital within the 5 years of follow-up, while $75 \%$ satisfied ACR classification criteria for RA by 5 years. The choice of relevant outcome measures is the subject of some debate. The development of radiological erosions is generally regarded as a reliable and validated measure of severity but, the earlier use of disease-modifying (DMARD) therapy may mean that erosions are of less clinical relevance. Furthermore, the level of disability experienced by patients is of far greater importance to them than $x$ ray appearances. Hence, a measure of physical function such as the HAQ score may provide a more relevant outcome measure. We have tested multiple measures of disease outcome but, despite this, we have found no association of the PTPN22*C1858T polymorphism with disease severity. One possibility is that the PTPN22*C1858T polymorphism is associated with outcome but this was masked by the effect of treatment. This could occur if patients carrying the variant allele were more likely to receive DMARD treatment. However, this was not found to be the case $(O R$ receiving DMARDs compared with not receiving DMARDs if carrying the PTPN221858*T variant $=1.36$ (95\% CI 0.84-2.24), $\mathrm{p}=0.19)$. 
Table 2 Genotype data for single nucleotide polymorphisms (SNPs) spanning the PTPN22 gene in patients with inflammatory polyarthritis (IP) and controls

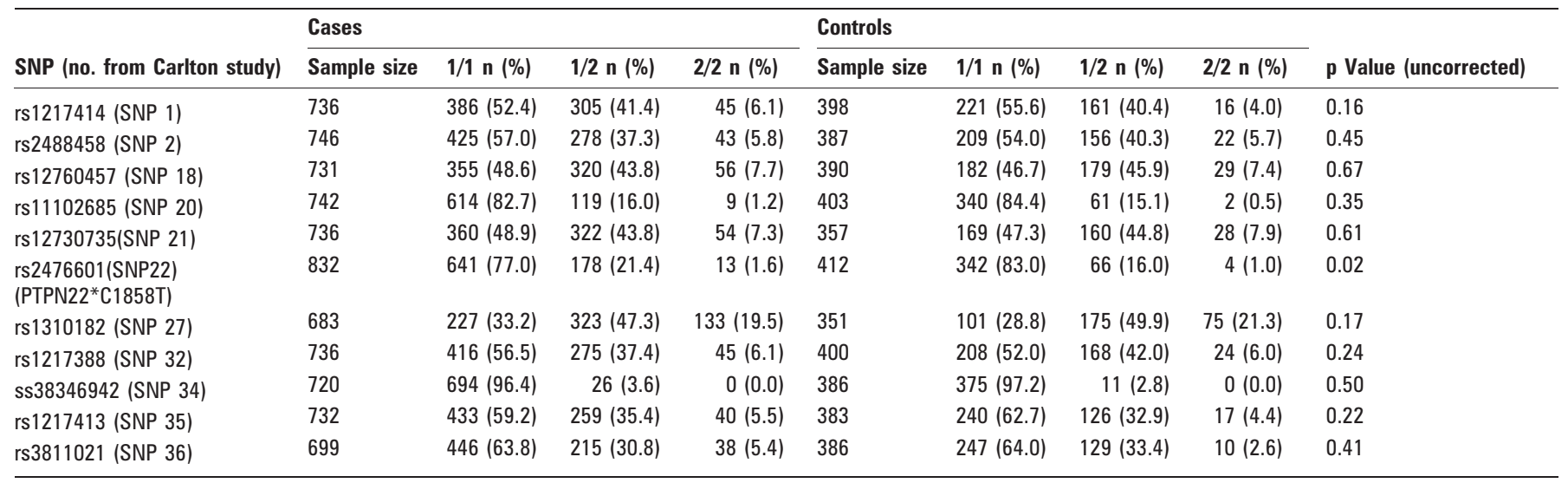

1, major (wild-type) allele; 2, minor (variant) allele.

Recently, Carlton et al reported that three SNPs (rs1310182 (SNP 27), rs3811021 (SNP 36) and rs3789604 (SNP 37)) were associated with susceptibility to RA independently of the PTPN22 *C1858T polymorphism. ${ }^{6}$ Their association with IP has not previously been determined. We did not test rs3789604 (SNP 37) in the current study but another SNP (rs3811021 (SNP 36)), which shows strong correlation with rs3789604, was examined. However, we found no association with susceptibility, either in IP as a whole or in the subgroup with RA, for any of the variants tested other than the PTPN22*C1858T polymorphism. Similarly, no evidence for association with severity measures was found, although it should be noted that the low minor allele frequencies for some of the SNP markers (particularly for ss38346944 (SNP 23), ss38346943 (SNP 28) and ss38346942 (SNP 34)) meant that power to test such association was limited.

Although the PTPN22*C1858T polymorphism was not associated with clinical markers of severity, in the subgroup of patients who fulfilled the ACR classification criteria for RA by 5 years, a statistically significant association with the presence of anti-CCP antibodies at baseline was detected, in keeping with previous reports. ${ }^{11} 20$ The presence of anti-CCP antibodies is strongly associated with adverse outcome. For example, the odds ratio for the presence of erosions by 2 years in patients who are anti-CCP antibody positive at baseline has been reported as 5.4 (95\% CI 1.7-17.0). ${ }^{21}$ Hence, although the PTPN22*1858T variant is associated with the presence of antiCCP antibody, it is the autoantibody that appears to determine severity. The findings are in keeping with the hypothesis that the PTPN22*1858T allele, like HLA-DRB1 shared epitope alleles, may create a permissive environment for the production of these antibodies. ${ }^{22}$

In summary, carriage of the PTPN22*1858T variant allele is associated with susceptibility both to IP and RA, conferring a modest increase in risk of approximately $40 \%$. The association appears to be solely accounted for by this SNP. Although the PTPN22*1858T variant allele was associated with the presence of anti-CCP autoantibodies, neither this nor other polymorphisms spanning the gene were found to be clinically useful predictors of adverse outcome.

Acknowledgements: We thank the Arthritis Research Campaign for their support.

Funding: ARC grant reference no. 17552.

Competing interests: None declared

Table 3 Allelic association of single nucleotide polymorphisms (SNPs) spanning PTPN22 gene with erosive status and Larsen score by 5 years

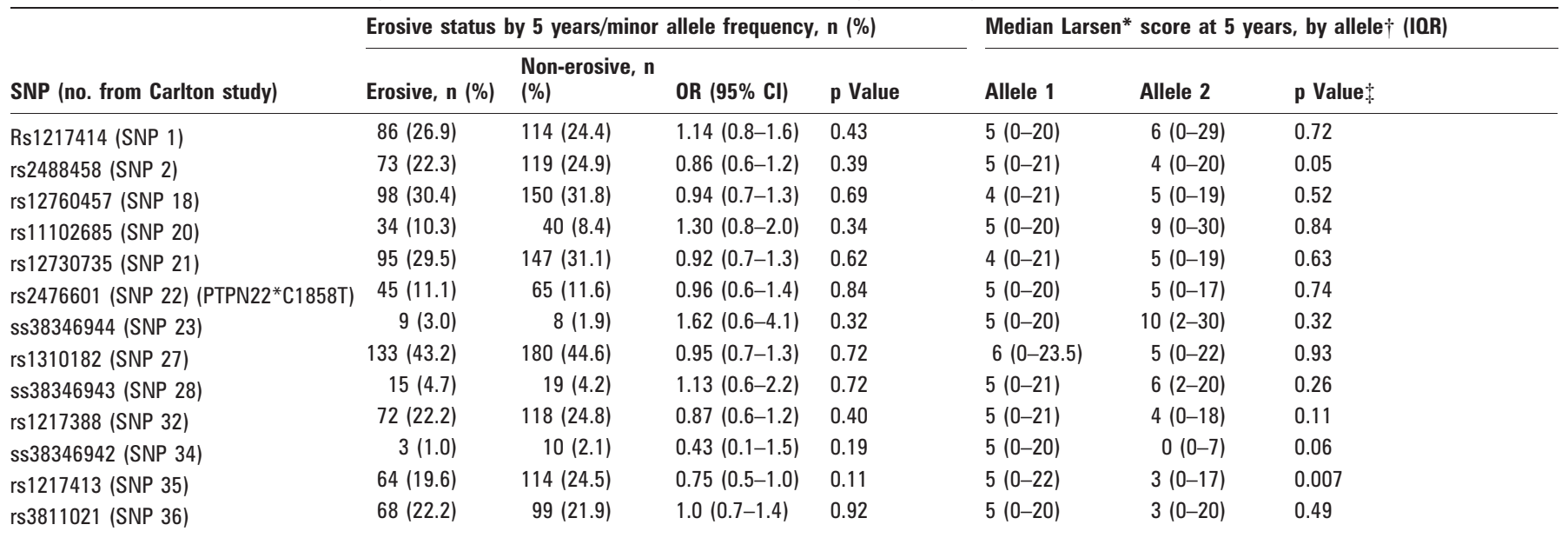

Uncorrected $\mathrm{p}$ values are presented. Allele 1, major (wild-type) allele; allele 2, minor (variant) allele.

*Larsen score in all subjects with information available. When the analysis was restricted to patients who were erosive, the conclusions were not altered; $\nmid \chi^{2}$; $₫$ logistic regression by genotype.

IQR, interquartile range; $\mathrm{OR}$, odds ratio. 
Table 4 Association of rs2476601 (PTPN22*C1858T) polymorphism with presence of autoantibodies in patients with inflammatory polyarthritis (IP) and in the subset satisfying ACR classification criteria for rheumatoid arthritis (RA)

\begin{tabular}{|c|c|c|c|c|}
\hline \multirow[b]{2}{*}{ Autoantibody at baseline } & \multicolumn{2}{|l|}{ IP Cohort } & \multicolumn{2}{|l|}{ RA subset } \\
\hline & PTPN22*CC, n (\%) & PTPN22*CT or PTPN22*TT, n (\%) & PTPN22*CC, n (\%) & PTPN22*CT or PTPN22*TT, n (\%) \\
\hline RF present & $185(74.0)$ & $65(26.0)$ & $155(74.5)$ & $53(25.5)$ \\
\hline RF absent & $442(78.2)$ & $123(21.8)$ & $231(79.4)$ & $60(20.6)$ \\
\hline Comparison: $p$ value & 0.19 & & 0.20 & \\
\hline Anti-CCP present & $109(68.5)$ & $50(31.5)$ & $102(69.4)$ & $45(30.6)$ \\
\hline Anti-CCP absent & $388(78.1)$ & $109(21.9)$ & $207(82.1)$ & $45(17.9)$ \\
\hline
\end{tabular}

Comparison: $\mathrm{p}$ value

0.003

$\mathrm{p}$ Values are uncorrected.

ACR, American College of Rheumatology; CCP, cyclic citrinulated peptide; RF, rheumatoid factor.

\section{REFERENCES}

1. MacGregor AJ, Snieder H, Rigby AS, Koskenvuo M, Kaprio J, Aho K, et al. Characterizing the quantitative genetic contribution to rheumatoid arthritis using data from twins. Arthritis Rheum 2000;43:30-7.

2. Orozco G, Rueda B, Martin J. Genetic basis of rheumatoid arthritis. Biomed Pharmacother 2006;60:656-62

3. Begovich AB, Carlton VE, Honigberg LA, Schrodi SJ, Chokkalingam AP, Alexander $\mathrm{HC}$, et al. A missense single-nucleotide polymorphism in a gene encoding a protein tyrosine phosphatase (PTPN22) is associated with rheumatoid arthritis. Am J Hum Genet 2004:75:330-7.

4. Hinks A, Worthington J, Thomson W. The association of PTPN22 with rheumatoid arthritis and juvenile idiopathic arthritis. Rheumatology (Oxford) 2006;45:365-8.

5. Vang T, Congia M, Macis MD, Musumeci L, Orru V, Zavattari $P$, et al. Autoimmuneassociated lymphoid tyrosine phosphatase is a gain-of-function variant. Nat Genet 2005:37:1317-19.

6. Carlton VE, Hu X, Chokkalingam AP, Schrodi SJ, Brandon R, Alexander HC, et al. PTPN22 genetic variation: evidence for multiple variants associated with rheumatoid arthritis. Am J Hum Genet 2005;77:567-81.

7. Hinks A, Eyre S, Barton A, Thomson W, Worthington J. Investigation of genetic variation across PTPN22 in UK rheumatoid arthritis (RA) patients. Ann Rheum Dis. 2006 Published Online First: 14 December 2006 doi:10.1136/ard.2006.060459

8. Wesoly J, Hu X, Thabet MM, Chang M, Uh H, Allaart CF, et al. The $620 \mathrm{~W}$ allele is the PTPN22 genetic variant conferring susceptibility to RA in a Dutch population. Rheumatology (Oxford) 2007; 46:617-21.

9. Thomson W, Harrison B, Ollier B, Wiles N, Payton T, Barrett J, et al. Quantifying the exact role of HLA-DRB1 alleles in susceptibility to inflammatory polyarthritis: results from a large, population-based study. Arthritis Rheum 1999;42:757-62.

10. Hinks A, Barton A, John S, Bruce I, Hawkins C, Griffiths CE, et al. Association between the PTPN22 gene and rheumatoid arthritis and juvenile idiopathic arthritis in a UK population: further support that PTPN22 is an autoimmunity gene. Arthritis Rheum 2005;52:1694-9.

11. Wesoly J, van der Helm-van Mil AH, Toes RE, Chokkalingam AP, Carlton VE, Begovich AB, et al. Association of the PTPN22 C1858T single-nucleotide polymorphism with rheumatoid arthritis phenotypes in an inception cohort. Arthritis Rheum 2005;52:2948-50.
12. Kirwan JR, Reeback JS. Stanford Health Assessment Questionnaire modified to assess disability in British patients with rheumatoid arthritis. Br J Rheumatol 1986:25:206-9.

13. Symmons DP, Silman AJ. The Norfolk Arthritis Register (NOAR). Clin Exp Rheumatol 2003;21(5 Suppl 31):S94-9.

14. Wiles N, Symmons DP, Harrison B, Barrett E, Barrett JH, Scott DG, et al. Estimating the incidence of rheumatoid arthritis: trying to hit a moving target? Arthritis Rheum 1999;42:1339-46

15. Bukhari M, Harrison B, Lunt M, Scott DG, Symmons DP, Silman AJ. Time to first occurrence of erosions in inflammatory polyarthritis: results from a prospective community-based study. Arthritis Rheum 2001;44:1248-53.

16. Larsen A, Dale K, Eek M. Radiographic evaluation of rheumatoid arthritis and related conditions by standard reference films. Acta Radiol Diagn (Stockh) 1977;18:481-91

17 Oeth P. Beaulieu M. Park C, Kosman D del Mistro G, van der Boom D et al. IPLEX assay: increased plexing efficiency and flexibility for MassARRAY ${ }^{\circledR}$ system through single base primer extension with mass-modified terminators. SEOUENOM ${ }^{\circledR}$ Application Update 2005; Apr 28. http://www.sequenom.com/Assets/pdfs/appnotes/ 8876-006.pdf (accessed 26 November 2007).

18. Barrett JC, Fry B, Maller J, Daly MJ. Haploview: analysis and visualization of LD and haplotype maps. Bioinformatics 2005;21:263-5.

19. Plenge RM, Padyukov L, Remmers EF, Purcell S, Lee AT, Karlson EW, et al. Replication of putative candidate-gene associations with rheumatoid arthritis in $>4,000$ samples from North America and Sweden: association of susceptibility with PTPN22, CTLA4, and PADI4. Am J Hum Genet 2005;77:1044-60.

20. Huizinga TW, Amos $\mathrm{Cl}$, van der Helm-van Mil AH, Chen W, van Gaalen FA, Jawaheer D, et al. Refining the complex rheumatoid arthritis phenotype based on specificity of the HLA-DRB1 shared epitope for antibodies to citrullinated proteins. Arthritis Rheum 2005:52:3433-8.

21. Berglin E, Johansson T, Sundin U, Jidell E, Wadell G, Hallmans G, et al. Radiological outcome in rheumatoid arthritis is predicted by presence of antibodies against cyclic citrullinated peptide before and at disease onset, and by IgA-RF at disease onset. Ann Rheum Dis 2006;65:453-8.

22. van der Helm-van Mil AH, Verpoort KN, Breedveld FC, Huizinga TW, Toes RE, de Vries RR. The HLA-DRB1 shared epitope alleles are primarily a risk factor for anticyclic citrullinated peptide antibodies and are not an independent risk factor for development of rheumatoid arthritis. Arthritis Rheum 2006;54:1117-21.

\section{Submit an eLetter, and join the debate}

eLetters are a fast and convenient way to register your opinion on topical and contentious medical issues. You can find the "submit a response" link alongside the abstract, full text and PDF versions of all our articles. We aim to publish swiftly, and your comments will be emailed directly to the author of the original article to allow them to respond. eLetters are a great way of participating in important clinical debates, so make sure your voice is heard. 